\title{
Teaching and Learning of Statistics
}

\author{
Dani Ben-Zvi and Katie Makar
}

\section{TSG-12 Rationale}

Being able to provide sound evidence-based arguments and critically evaluate data-based claims are important skills that all citizens should have. It is not surprising therefore that the study of statistics at all educational levels is gaining more students and drawing more attention than it has in the past. The study of statistics provides students with tools, ideas and dispositions to use in order to react intelligently to information in the world around them. Reflecting this need to improve students' ability to think statistically, statistical literacy and reasoning are becoming part of the mainstream school and university curriculum in many countries.

As a consequence, statistics education is a growing and becoming an exciting field of research and development. Statistics at school level is usually taught in the mathematics classroom in connection with learning probability. Topic Study Group 12 (TSG-12) included probabilistic aspects in learning statistics, whereas research with a specific focus on learning probability was discussed in TSG-11 of ICME-12.

Organizers Co-chairs: Dani Ben-Zvi (Israel), Katie Makar (Australia); Team Members: Lisbeth Cordani (Brazil), Arthur Bakker (The Netherlands), Jangsun Paek (Korea); Liaison IPC Member: Gail Burrill (USA).

D. Ben-Zvi (ه)

University of Haifa, Haifa, Israel

e-mail: dbenzvi@univ.haifa.ac.il

K. Makar

University of Queensland, Queensland, Australia

e-mail: k.makar@uq.edu.au

(C) The Author(s) 2015 


\section{TSG-12 Meetings During ICME-12}

This growing interest in statistics education was reflected in the popularity of this group and in the more than 40 papers accepted for presentation. The members of TSG-12 came from twenty different countries and varied significantly by experience, background and seniority. The presentations were divided into six themes of key issues in statistics education research: (a) theoretical issues in learning statistics; (b) integrating statistics with students' experiences; (c) the emergence of students' statistical reasoning; (d) teachers' statistical knowledge and learning and professional development of teachers; (e) learning technology in statistics education; and (f) learning statistics in school and at the tertiary level.

The four meetings of TSG-12 were organized to create a sense of community among all presenters and participants, who shared a common desire to improve statistics education by focusing on conceptual understanding rather than rote learning. To build and support this sense of community we asked participants to prepare for TSG-12 before they arrived in Seoul by reading all papers in advance, so we could discuss each other's work; the co-chairs kept informal correspondence with all participants before, during and after the conference; and finally, participants were asked to be involved every day of the program so we could get to know one another, develop collegial networks, welcome our emerging scholars and discuss the important work in statistics education research around the world.

Because of the large number of proposals we received, the time available only allowed for relatively short presentations by the authors. However, we felt it critical that all proposals be given time for presentation in some format. The four meetings were therefore organized to capitalize on community-building and discussions around our collective and individual research. Some of the sessions ran in parallel, some in roundtable format. While there was a poster session which is common for all TSGs, half of one TSG-12 session was dedicated to poster presentations so that the TSG-12 community could engage more directly with their authors and each other in a relaxed setting. Another highlight of the program was a panel of discussants on the final day to reflect as a community on the themes, presentations, issues raised and discussions over the four days.

The accepted papers were organized in the following ways:

- About twenty poster presentations to engage TSG-12 community discussions with diverse and thought-provoking studies;

- Eleven short presentations $(5+5 \mathrm{~min}$ discussion) in a roundtable format organized into four themes to enrich understanding of the themes and allow for extended discussions around common interests;

- Twelve longer presentations and discussions $(10+5$ min discussion $)$ to enhance the overarching themes of the short presentation and poster sessions;

- Four major long presentations $(20+10$ min discussion) to provoke initial discussions and stimulate final day reflections among the whole TSG-12 community. These papers were authored by Andreas Eichler and Markus Vogel 
(Germany), Arthur Bakker, Xaviera van Mierlo and Sanne Akkerman (The Netherlands); Luis Saldanha and Michael McAllister (USA); and Dani Ben-Zvi and Keren Aridor-Berger (Israel).

\section{TSG-12 Beyond the Conference}

Informal feedback received after the conference was extremely positive. We felt at the end that much can be learned by integrating results from such a variety of research and practice in statistics education. This integration of theories, empirical evidence and instructional methods can eventually help students to develop their statistical thinking. These ongoing efforts to reform statistics instruction and content have the potential to both make the learning of statistics more engaging and prepare a generation of future citizens that deeply understand the rationale, perspective and key ideas of statistics. These are skills and knowledge that are crucial in the current age of information.

An informal set of proceedings was created to allow for immediate distribution of the TSG-12 papers among those within and beyond the TSG-12 members. The proceedings are available at: http://dbz.edtech.haifa.ac.il/publications/books. Many of the members of the community that came together for TSG-12 have remained in touch through a sharing of contact details and plans to meet again at the Ninth International Conference on Teaching Statistics (ICOTS-9) in 2014. Based on the TSG-12 papers, the book Teaching and learning of statistics: International perspectives, edited by Ben-Zvi and Makar, was published in 2014 by the Statistics Education Center, the University of Haifa, Israel.

\section{TSG-12 Organizing Team}

\begin{tabular}{l|l|l}
\hline \multirow{2}{*}{ Co-chairs } & Dani Ben-Zvi (Israel) & dbenzvi@univ.haifa.ac.il \\
\cline { 2 - 3 } & Katie Makar (Australia) & k.makar@uq.edu.au \\
\hline \multirow{2}{*}{ Team members } & Jangsun Baek (Korea) & jbaek@jnu.ac.kr \\
\cline { 2 - 3 } & Arthur Bakker (The Netherlands) & a.bakker4@uu.nl \\
\cline { 2 - 3 } & Lisbeth Cordani (Brazil) & lisbeth@ime.usp.br \\
\hline Liaison IPC member & Gail Burrill (USA) & burrill@msu.edu \\
\hline
\end{tabular}

\section{TSG-12 Resources}

- TSG-12 Website: http://www.icme12.org/sub/tsg/tsgload.asp?tsgNo=12.

- TSG-12 Proceedings (eBook): http://dbz.edtech.haifa.ac.il/publications/books. 
Open Access This chapter is distributed under the terms of the Creative Commons Attribution Noncommercial License, which permits any noncommercial use, distribution, and reproduction in any medium, provided the original author(s) and source are credited. 